\title{
ON THE BOREL SUMMABILITY OF DIVERGENT SOLUTIONS OF THE HEAT EQUATION
}

\author{
D. A. LUTZ, M. MIYAKE AND R. SCHÄFKE
}

\begin{abstract}
In recent years, the theory of Borel summability or multisummability of divergent power series of one variable has been established and it has been proved that every formal solution of an ordinary differential equation with irregular singular point is multisummable. For partial differential equations the summability problem for divergent solutions has not been studied so well, and in this paper we shall try to develop the Borel summability of divergent solutions of the Cauchy problem of the complex heat equation, since the heat equation is a typical and an important equation where we meet diveregent solutions. In conclusion, the Borel summability of a formal solution is characterized by an analytic continuation property together with its growth condition of Cauchy data to infinity along a stripe domain, and the Borel sum is nothing but the solution given by the integral expression by the heat kernel. We also give new ways to get the heat kernel from the Borel sum by taking a special Cauchy data.
\end{abstract}

\section{$\S 0$. Introduction and main results}

We consider the following "characteristic" Cauchy problem for the complex heat equation

$$
\frac{\partial}{\partial \tau} u(\tau, z)=\frac{\partial^{2}}{\partial z^{2}} u(\tau, z), \quad u(0, z)=\varphi(z)
$$

where $\tau=t+i \eta$ and $z=x+i y$ are complex variables and $\varphi(z)$ is assumed to be analytic in a domain $D$, which we assume to contain a neighborhood of the origin without loss of generality.

As easily seen, this Cauchy problem has the unique formal solution

$$
\hat{u}(\tau, z)=\sum_{n=0}^{\infty} \varphi^{(2 n)}(z) \frac{\tau^{n}}{n !} \underset{\text { put }}{=} \sum_{n=0}^{\infty} u_{n}(z) \tau^{n},
$$

where $\varphi^{(k)}=d^{k} \varphi / d z^{k}(k \geq 0)$. This formal solution diverges for general Cauchy data $\varphi(z)$, as is shown by the example $\varphi(z)=(1-z)^{-1}$, where $u_{n}(z)=\frac{(2 n !)}{n !}(1-z)^{-2 n-1}$.

Received July 26, 1996. 
By Cauchy's integral formula, for any $r>0$ such that $\{|z| \leq r\} \subset$ $D$, we have the following estimate for $\left\{u_{n}(z)\right\}_{n=0}^{\infty}$ by taking some positive constants $A$ and $B$,

$$
\max _{|z| \leq r}\left|u_{n}(z)\right| \leq A B^{n} n !, \quad \text { for all } n=0,1,2, \ldots
$$

Such a formal power series is called Gevrey of order one in the variable $\tau$.

In the one variable case of ordinary differential equations, after the fundamental work of J. Ecalle, great progress has been made in the theory of summability for Gevrey type power series by many authors. As an application, they proved that every formal solution of an analytic ordinary differential equation is multi-summable (cf. [Ram 1], [Mal], [Bal] and references cited there).

For partial differential equations, a corresponding theory has been slower to develop. See recent work by [Ger-Tah], [Miy 2], [Miy 3], [Miy-Has], [Miy-Yos 1], [Yon] on the Gevrey properties of formal solutions and by S. Ouchi [Ōuc] on an asymptotic interpretation of formal solutions. Our purpose in this paper is to improve the asymptotic results by discussing the summation of formal solutions and under which conditions this is possible or not possible. While some of the results could possibly be extended to more general differential equations, we choose to consider only the heat equation since in this simple case we obtain both necessary and sufficient conditions.

It is natural to ask the following questions about the formal solution $\hat{u}(\tau, z)$ constructed above:

[1] under what conditions on $\varphi(z)$ does the formal solution (0.2) converge?

[2] if (0.2) diverges, is it an asymptotic expansion of actual solutions as $\tau \rightarrow 0$ in sectorial domains?

[3] if asymptotic solutions exist, when are they unique and how can they be constructed?

As regards question [1], an easy way to get a sufficient condition for (0.2) to converges is to assume the Cauchy data $\varphi(z)$ satisfy the following inequalities for some positive constants $r, C$ and $D$

$$
\max _{|z| \leq r}\left|\varphi^{(n)}(z)\right| \leq C D^{n}(n !)^{1 / 2}, \quad \text { for all } n=0,1,2, \ldots
$$


This condition is equivalent to assuming $\varphi(z)$ to be an entire function of exponential order at most 2 , that is, the following inequality holds for some positive constants $E$ and $\delta$,

$$
|\varphi(z)| \leq E e^{\delta|z|^{2}}, \quad z \in \mathbb{C}
$$

It is remarkable that S. Kowalevskaya already proved in her historical paper on the Cauchy problem for analytic partial differential equations published in 1875 that these conditions are also necessary for the convergence of the formal solution (0.2) ([Kow], see also [Pic]). Thus we know a complete answer to the question [1]. Kowalevskaya's result tells us that there can not exist any locally analytic solutions of the heat equation in the variable $z$. Precisely, they should be entire functions in the $z$ variable with an exponential growth condition of order at most 2 .

On the question [2], S. Öuchi studied divergent formal solutions of the characteristic Cauchy problem for partial differential equations, and under fairly general conditions for the operators he proved that the formal solutions are asymptotic expansions (in the sense of Poincaré) of actual solutions as $\tau \rightarrow 0$ in sectorial domains with small opening angle ([Ōuc]). In this sense, we have a satisfactory answer to the question [2].

His result is stated as follows in our case:

Let $S$ be a sectorial domain of opening angle $\alpha$ less than $\pi$ with the origin as its vertex (the radius is assumed to be small). Then there exists an analytic solution $u(\tau, z)$ of the heat equation defined on $S \times\{|z| \leq r\}$ for small $r>0$ whose asymptotic expansion (in the sense of Poincaré) as $S \ni \tau \rightarrow 0$ is $\hat{u}(\tau, z)$, i.e., satisfying

$$
\max _{|z| \leq r}\left|u(\tau, z)-\sum_{n=0}^{N} u_{n}(z) \tau^{n}\right|=o\left(|\tau|^{N}\right), \quad \text { as } \tau \rightarrow 0(\tau \in S)
$$

for all $N=0,1,2, \ldots$.

Unfortunately, there are infinitely many such actual solutions with $\hat{u}(\tau, z)$ as their asymptotic expansion in any sector of opening angle less than $\pi$ (see Example in Section 2).

Our main interest in this paper concerns answers to question [3] in the cases where the angle opening $\alpha$ of an "asymptotic sector" is at least $\pi$. Here, the concepts of Borel summability and fine Borel summability are important. 
Postponing the exact definitions in our situation to the next section, we state our main results as follows:

(A) The formal solution $\hat{u}(\tau, z)$ is Borel summable to an actual solution in a sector of the complex $\tau$-plane in the direction $\theta$ and of opening angle $\alpha>\pi$ if and only if the Cauchy data $\varphi(z)$ is analytic in a domain containing a double cone

$$
D(\theta, \alpha)=S\left(\frac{\theta}{2}, \frac{\alpha-\pi}{2}, \infty\right) \cup S\left(\pi+\frac{\theta}{2}, \frac{\alpha-\pi}{2}, \infty\right)
$$

and has at most exponential growth order two as $|z| \rightarrow \infty$ there.

(B) the formal solution $\hat{u}(\tau, z)$ is finely Borel summable in a direction $\theta$, if and only if the Cauchy data $\varphi(z)$ is analytic in a strip

$$
\Omega\left(\frac{\theta}{2}, \omega\right)=\left\{z \in \mathbb{C} \mid \operatorname{dist}\left(z, e^{i \theta / 2} \mathbb{R}\right)<\omega\right\}
$$

for some $\omega>0$ and has at most exponential growth order two as $|z| \rightarrow 0$ there.

It follows from our results, that just the local analyticity of the Cauchy data is insufficient for either Borel summability or fine Borel summability, to hold even though these are in some sense locally defined concepts. This is somewhat surprising, but also to be expected in view of Kowalevskaya's result.

In Section 1, we shall give a summary of the theory of Gevrey asymptotic expansions and Borel summability without proofs, since they are essentially the same as in the one variable case. The most important idea here is a transfer principle between the Borel summability of $\hat{u}$ and properties of its Borel associated function.

In Section 2, we shall give a refined form of Ōuchi's result for the case of Gevrey asymptotic expansion in sectors of opening angle less than $\pi$.

In Section 3, we shall prove the main theorems and give more precise properties for the fine Borel sum of the formal solution. As a corollary we shall give a characterization of Borel summability in sectors of opening angle larger than $\pi$, where we shall meet again Kowalevskaya's result if we ask for the validly of the asymptotic expansion of the Borel sum in sectors with opening angle larger than $3 \pi$. The main results follow from a complete correspondance between the properties of the Borel associated function and the Cauchy data. 
In Section 4, we apply the result to convergence of factorial series expansions for the fine Borel sum, and give a recursion formula for the coefficients of a factorial series expansion.

In Section 5, we shall present new derivations of the heat kernel by using the Borel sum of the formal solution with the Cauchy data $\varphi(z)=1 / z$.

Acknowledgements. The authors first discussed problems of this type for the heat equation during their stay at the I.R.M.A., Université Louis Pasteur, Strasbourg in June of 1993 and wish to give special thanks to Professor Raymond Gérard for making this possible.

\section{$\S 1$. Summary of Gevrey asymptotic expansions}

We summarize the fundamentals on Gevrey asymptotic expansions and the Borel summability for Gevrey type formal power series without the proofs, since they are essentially the same with one variable case studied by many authors (see, [Ram 2], [Mal], [Bal]). In fact, the only difference with them is that we consider the formal power series over the ring of analytic functions, not over just the complex numbers.

\subsection{Formal power series}

$\mathcal{O}(r)$ is the ring of analytic functions on $B(r):=\{z \in \mathbb{C}|| z \mid \leq r\}$. $\mathcal{O}(r)[[\tau]]$ is the ring of formal power series in $\tau$ over the ring $\mathcal{O}(r)$, and we define $\mathcal{O}[[\tau]]$ by

$$
\mathcal{O}[[\tau]]:=\bigcup_{r>0} \mathcal{O}(r)[[\tau]] .
$$

An element $\hat{u}(\tau, z) \in \mathcal{O}[[\tau]]$ is written as

$$
\hat{u}(\tau, z)=\sum_{n=0}^{\infty} u_{n}(z) \tau^{n}, \quad u_{n}(z) \in \mathcal{O}(r) \text { for some } r>0 .
$$

\subsection{1-Gevrey formal power series}

$\mathcal{O}(r)[[\tau]]_{1}$, which is called of class Gevrey one or 1-Gevrey for short, is the subring of $\mathcal{O}(r)[[\tau]]$ whose coefficient satisfy the following inequalities for some positive constants $C$ and $K$,

$$
\max _{|z| \leq r}\left|u_{n}(z)\right| \leq C K^{n} n !, \quad \text { for } n=0,1,2, \ldots
$$

Also we define $\mathcal{O}[[\tau]]_{1}$ by

$$
\mathcal{O}[[\tau]]_{1}:=\bigcup_{r>0} \mathcal{O}(r)[[\tau]]_{1}
$$




\section{3.}

For $\theta \in \mathbb{R}, \alpha>0$ and $0<T \leq+\infty$, we denote by $S(\theta, \alpha ; T)$ a sectorial domain defined by

$$
S(\theta, \alpha ; T):=\{\tau \in \mathbb{C}|| \arg (\tau)-\theta|<\alpha / 2,0<| r \mid<T\} .
$$

Here $\theta, \alpha$ and $T$ are called the direction, opening angle, and radius of the sectorial domain $S(\theta, \alpha ; T)$, respectively. If the radius $T$ is not so important to identify, we will sometimes suppress it and denote the sector by $S(\theta, \alpha)$ for simplicity. A sectorial domain $S^{\prime}$ is called a proper subsector of $S(\theta, \alpha ; T)$ if its closure is contained in $S(\theta, \alpha ; T) \cup\{0\}$.

\subsection{Gevrey asymptotic expansion}

Let $u(\tau, z)$ be analytic on $\bigcap_{\alpha^{\prime}<\alpha} S\left(\theta, \alpha^{\prime}\right) \times B\left(r\left(\alpha^{\prime}\right)\right)$, where $r\left(\alpha^{\prime}\right)$ may tend to 0 as $\alpha^{\prime} \rightarrow \alpha$. Then $\hat{u}(\tau, z) \in \mathcal{O}[[t]]_{1}$ is called a Gevrey asymptotic expansion of $u(\tau, z)$ as $\tau \rightarrow 0$ in $S(\theta, \alpha)$ or shortly in $S(\theta, \alpha)$ if for any proper subsector $S^{\prime} \subset S(\theta, \alpha ; T)$ (with sufficiently small radius), there exist positive constants $C, K$ and $0<r_{1}<r$ such that $\hat{u}(\tau, z) \in \mathcal{O}\left(r_{1}\right)[[\tau]]_{1}$ and

$$
\begin{aligned}
\max _{|z| \leq r_{1}}\left|u(\tau, z)-\sum_{n=0}^{N-1} u_{n}(z) \tau^{n}\right| \leq & C K^{N} N !|\tau|^{N}, \\
& \tau \in S^{\prime}, N=1,2,3, \ldots
\end{aligned}
$$

This relation is denoted by

$$
u(\tau, z) \sim_{1} \hat{u}(\tau, z), \quad \text { in } S(\theta, \alpha) .
$$

An analytic function $u(\tau, z)$ is said to be Gevrey asymptotic expandable in $S(\theta, \alpha)$ if it has a Gevrey asymptotic expansion $\hat{u}(\tau, z) \in \mathcal{O}[[\tau]]_{1}$.

Let us denote by $\mathcal{A}^{(1)}(S(\theta, \alpha))$ the set of analytic functions which are Gevrey asymptotic expandable in $S(\theta, \alpha)$. We define a mapping $J^{(1)}$ by

$$
J^{(1)}: \mathcal{A}^{(1)}(S(\theta, \alpha)) \longrightarrow \mathcal{O}[[\tau]]_{1},
$$

where $J^{(1)}(u(\tau, z))=\hat{u}(\tau, z)$ is the Gevrey asymptotic expansion $\hat{u}(\tau, z)$ of $u(\tau, z)$.

Now the following result is known as an analogue of Borel-Ritt's theorem for Gevrey asymptotic expansions (cf. [Ram 2], [Mal], [Bal]).

THEOREM 1.1. (1) The mapping $J^{(1)}$ defined by (1.8) is surjective but is not injective for any $\theta \in \mathbb{R}$ and $\alpha$ with $\alpha \leq \pi$.

(2) For any $\alpha$ with $\alpha>\pi$, the mapping $J^{(1)}$ is not surjective but is injective for any $\theta \in \mathbb{R}$. 


\subsection{Formal Borel transform}

To get an element $u(\tau, z) \in\left(J^{(1)}\right)^{-1}(\hat{u})$ for $\hat{u} \in \mathcal{O}[[\tau]]_{1}$, an effective way is to introduce the formal Borel transform $v(s, z)$ of $\hat{u}(\tau, z)$ defined by

$$
v(s, z):=\sum_{n=0}^{\infty} u_{n}(z) \frac{s^{n}}{n !} .
$$

By the definition of $\mathcal{O}[[\tau]]_{1}, v(s, z)$ is analytic in a neighborhood of the origin $(s, z)=(0,0)$, and so we assume that it is analytic on $\{|s|<r\} \times\{|z|<r\}$ $\left({ }^{\exists} r>0\right)$. Now for any $\theta \in \mathbb{R}$, we fix a positive constant $\rho$ such that $0<\rho<$ $r$, and we define

$$
u^{(\theta, \rho)}(\tau, z):=\frac{1}{\tau} \int_{0}^{\rho e^{i \theta}} e^{-s / \tau} v(s, z) d s, \quad|\arg (\tau)-\theta|<\pi / 2 .
$$

Then it can be proved ([Bal]) that $J^{(1)}\left(u^{(\theta, \rho)}(\tau, z)\right)=\hat{u}(\tau, z)$ in $S(\theta, \pi)$. Here, the arbitrariness of $\rho$ shows the non uniqueness of functions $u(\tau, z)$ such that $J^{(1)}(u)=\hat{u}$ in $S(\theta, \pi)$.

\subsection{Borel summability}

According to the assertion (2) of theorem 1.1, we know that if the opening angle of a sector $S$ is larger than $\pi$, for $\hat{u}(\tau, z) \in J^{(1)}\left(\mathcal{A}^{(1)}(S)\right.$ ), $u(\tau, z) \in \mathcal{A}^{(1)}(S)$ such that $J^{(1)}(u)=\hat{u}$ is unique. This $u(\tau, z)$ is called the Borel sum of $\hat{u}(\tau, z)$, and $\hat{u}(\tau, z)$ is said to be Borel summable in $S$. The Borel summability of $\hat{u}(\tau, z) \in \mathcal{O}[[\tau]]_{1}$ can be characterized (with respect to its formal Borel transform) as follows:

TheOrem 1.2. A formal series $\hat{u}(\tau, z) \in \mathcal{O}[[\tau]]_{1}$ is Borel summable in $S(\theta, \alpha)(\alpha>\pi)$ if and only if its Borel transform $v(s, z)$ is analytic in $S(\theta, \alpha-\pi ; \infty) \times B(r)$ and satisfies a growth condition of exponential type as $s \rightarrow \infty$ in $S(\theta, \alpha-\pi ; \infty)$, i.e., for any proper subsector $S^{\prime} \subset S(\theta, \alpha-\pi ; \infty)$ of infinite radius there exists $0<r_{1}<r$ such that

$$
\max _{|z| \leq r_{1}}|v(s, z)| \leq C e^{\delta|s|}, \quad s \in S^{\prime}
$$

for some constants $C$ and $\delta$. Finally, the Borel sum $\left(J^{(1)}\right)^{-1}(\hat{u})$ in $S(\theta, \alpha)$ is represented by the Laplace integral,

$$
u^{\varphi}(\tau, z)=\frac{1}{\tau} \int_{0}^{\infty e^{i \varphi}} e^{-s / \tau} v(s, z) d s
$$

where the integration in taken over the ray $e^{i \varphi} \mathbb{R}_{+}=\left\{r e^{i \varphi} \mid r \geq 0\right\}\left(\mathbb{R}_{+}:=\right.$ $[0, \infty))$ for $\varphi$ such that $|\varphi-\theta|<\alpha-\pi$. 


\subsection{Fine Borel summability}

In the definition of Gevrey asymptotic expansion (1.6), we always take a proper subsector $S^{\prime} \subset S(\theta, \alpha)$. In the case of crucial value of $\alpha=\pi, \mathrm{F}$. Nevanlinna [Nev] have gave a refined form of asymptotic expansion which corresponds to taking open disks instead of subsectors as follows. For $\theta \in \mathbb{R}$ and $T>0$ we define an open disk $O(\theta, T)$ by

$$
O(\theta, T):=\left\{\tau \in \mathbb{C}|| \tau-T e^{i \theta} \mid<T\right\} .
$$

Then we say that $\hat{u}(\tau, z) \in \mathcal{O}[[\tau]]_{1}$ is finely Borel summable in a direction $\theta$ if there exists an analytic function $u(\tau, z)$ in $O(\theta, T) \times B(r)$ $\left({ }^{\exists} T,{ }^{\exists} r>0\right)$ such that for some $0<T^{\prime}<T$ the following inequalities

$$
\begin{aligned}
\max _{|z| \leq r}\left|u(\tau, z)-\sum_{n=0}^{N-1} u_{n}(z) \tau^{n}\right| \leq & C K^{N} N !|\tau|^{N} \\
& N=1,2,3, \ldots ; \tau \in O\left(\theta, T^{\prime}\right)
\end{aligned}
$$

hold for some positive constants $C$ and $K$. This relation is denoted by

$$
u(\tau, z) \sim_{1} \hat{u}(\tau, z), \quad \text { finely in the direction } \theta .
$$

For a modern treatment of fine Borel summability, see Malgrange [Mal].

As in the Borel summable case, it is proved that if $u(\tau, z) \sim_{1} 0$ finely in $\theta$ we have $u(\tau, z) \equiv 0$ ([Mal $])$. Hence for $\hat{u}(\tau, z) \in \mathcal{O}[[\tau]]_{1}$, if there exists $u(\tau, z)$ such that the relation (1.15) holds, it is called the fine Borel sum in $\theta$ direction of $\hat{u}(\tau, z)$ (see [Mal]).

To characterize the fine Borel summability we need to define a set $E_{+}(\theta, w)$ by

$$
E_{+}(\theta, w):=\left\{s \in \mathbb{C} \mid \operatorname{dist}\left(s, e^{i \theta} \mathbb{R}_{+}\right)<w\right\} .
$$

Now the fine summability is characterized as follows.

THEOREM 1.3. The formal power series $\hat{u}(\tau, z) \in \mathcal{O}[[\tau]]_{1}$ is fine Borel summable in a direction $\theta$ if and only if its formal Borel transform $v(s, z)$ is analytic on $E_{+}(\theta, w) \times B(r)$ for some $w>0$ and $r>0$, and satisfies $a$ growth condition of exponential type as $s \rightarrow \infty$ in $E_{+}(\theta, w)$, that is,

$$
\max _{|z| \leq r}|v(s, z)| \leq C e^{\delta|s|}, \quad s \in E_{+}(\theta, w) .
$$

The fine Borel sum $u^{\theta}(\tau, z)$ in the direction $\theta$ of $\hat{u}(\tau, z)$ is obtained by the expression (1.12).

For the proof, see Malgrange [Mal]. 


\subsection{Functions satisfying $J^{(1)}(u)=0$}

The following criterion for analytic functions with 0 Gevrey asymptotic expansion is used in the following sections.

Proposition 1.4. In order that $u(\tau, z) \sim_{1} 0$ in $S(\theta, \alpha)$ it is necessary and sufficient that for any proper subsector $S^{\prime} \subset S(\theta, \alpha)$ there exist positive constants $r_{1}, C$ and $\delta$ such that

$$
\max _{|z| \leq r_{1}}|u(\tau, z)| \leq C e^{-\delta /|\tau|}, \quad \tau \in S^{\prime}
$$

In the case of fine summability in a direction $\theta, S^{\prime}$ should be replaced by $O(\theta, T)$ for some $T>0$, and the ineqiality implies that $u \equiv 0$ by Watson's lemma (cf. [Mal]).

\section{$\S 2$. Existence of actual solutions in small sectors}

By Theorem 1.1 we know that the mapping $J^{(1)}: \mathcal{A}(S(\theta, \alpha)) \rightarrow \mathcal{O}[[\tau]]_{1}$ is surjective whenever $\alpha \leq \pi$ for any $\theta \in \mathbb{R}$. Corresponding to this result we can prove the similar result for the formal solution (0.2) of the Cauchy problem (0.1) which is the Gevrey version of Ouchi's result stated in the Introduction.

THEOREM 2.1. Let $\hat{u}(\tau, z)$ be the formal solution (0.2) where $\varphi(z)$ is assumed to be just analytic at 0 . Then for any $\theta \in \mathbb{R}$ and any $\alpha$ with $0<\alpha \leq \pi$, there exists an actual solution $u(\tau, z)$ of the heat equation such that $u(\tau, z) \sim_{1} \hat{u}(\tau, z)$ in $S(\theta, \alpha)$. Moreover, there are infinitely many such solutions.

Let $\hat{u}(\tau, z)$ be the formal solution (0.2). By Theorem 1.1, there exists $u(\tau, z)$ analytic on $S(\theta, \pi ; T) \times B(r)$ such that $u(\tau, z) \sim_{1} \hat{u}(\tau, z)$ in $S(\theta, \pi)$ by taking small $T$ and $r$. Then it holds that

$$
\frac{\partial}{\partial \tau} u(\tau, z)-\frac{\partial^{2}}{\partial z^{2}} u(\tau, z) \underset{\text { put }}{=} f(\tau, z) \sim_{1} 0 \quad \text { in } S(\theta, \pi)
$$

Now the first part of Theorem 2.1 is an immediate consequence of the following.

LEMma 2.2. Let $f(\tau, z) \sim_{1} 0$ in $S(\theta, \alpha)$ with $\alpha \leq \pi$. Then for any $\psi_{i}(\tau) \sim_{1} 0$ in $S(\theta, \alpha)(i=0,1)$, the Cauchy problem

$$
\frac{\partial^{2}}{\partial z^{2}} w(\tau, z)-\frac{\partial}{\partial \tau} w(\tau, z)=f(\tau, z), \quad \frac{\partial^{i} w}{\partial z^{i}}(\tau, 0)=\psi_{i}(\tau) \quad(i=0,1)
$$

has a unique solution $w(\tau, z)$ satisfying $w(\tau, z) \sim_{1} 0$ in $S(\theta, \alpha)$. 
Proof. By changing the unknown function $w(\tau, z)$ by $w(\tau, z)-\psi_{0}(\tau)-$ $\psi_{1}(\tau) z$, we may assume that $\psi_{i}(\tau) \equiv 0(i=0,1)$ for the Cauchy data. For any $\beta$ with $0<\beta<\alpha$, by taking small $T$ and $r$, we may assume that $f(\tau, z)$ is analytic on $S(\theta, \beta ; T) \times B(r)$. Then the unique existence of an analytic solution $w(\tau, z)$ on $S(\theta, \beta ; T) \times B(r)$ is known from the general theory (cf. M. Miyake [Miy 2, theorem 3.1]). Therefore we have to prove that $w(\tau, z) \sim_{1} 0$ in $S(\theta, \beta)$. From Proposition 1.4, it is sufficient to prove that for any positive constant $\alpha^{\prime}$ with $\alpha^{\prime}<\beta$, there exists a positive constant $r_{1}$ such that

$$
\max _{|z| \leq r_{1}}|w(\tau, z)| \leq C e^{-\delta /|\tau|}, \quad \tau \in S\left(\theta, \alpha^{\prime}\right)
$$

holds for some positive constants $C$ and $\delta$.

Since $f(\tau, z) \sim_{1} 0$ in $S(\theta, \alpha)$ we may assume

$$
\max _{|z| \leq r}|f(\tau, z)| \leq A e^{-c /|\tau|}, \quad \tau \in S(\theta, \beta) \quad(\text { because } \beta<\alpha),
$$

for some positive constants $A$ and $c$. For simplicity in the proof below, we assume that the direction $\theta=0$ without loss of generality. Let

$$
f(\tau, z)=\sum_{n=0}^{\infty} f_{n}(\tau) \frac{z^{n}}{n !}
$$

be the Taylor expansion of $f(\tau, z)$. Then an easy calculation shows the following Taylor expansion for $w(\tau, z)$,

$$
\begin{aligned}
& w(\tau, z)= \sum_{n=1}^{\infty} \frac{z^{2 n}}{(2 n) !}\left(\sum_{k=1}^{n} \frac{d^{k-1}}{d \tau^{k-1}} f_{2(n-k)}(\tau)\right) \\
&+\sum_{n=1}^{\infty} \frac{z^{2 n+1}}{(2 n+1) !}\left(\sum_{k=1}^{n} \frac{d^{k-1}}{d \tau^{k-1}} f_{2(n-k)+1}(\tau)\right) \\
& \underset{\text { put }}{=} w_{+}(\tau, z)+w_{-}(\tau, z) .
\end{aligned}
$$

In order to prove the estimate $(2.2)$ for $w(\tau, z)$, it is sufficient to consider the part $w_{+}(\tau, z)$, since the other part is similar.

We choose and fix a positive constant $\varepsilon$ satisfying $\alpha^{\prime}+\varepsilon<\beta$. For $\tau \in$ $S\left(0, \alpha^{\prime} ; T / 2\right)$, we choose a closed curve $\Gamma_{\tau, \varepsilon}:=\partial\left\{S\left(0, \alpha^{\prime}+\varepsilon ; 2|\tau|\right) \backslash S\left(0, \alpha^{\prime}+\right.\right.$ $\varepsilon ;|\tau| / 2)\}$, where $\partial \Omega$ denotes the boundary of domain $\Omega$. Then for $\tau \in$ 
$S\left(0, \alpha^{\prime} ; T / 2\right)$ Cauchy's integral formula implies

$$
\frac{d^{k-1}}{d \tau^{k-1}} f_{2(n-k)}(\tau)=\frac{\{2(n-k)\} !(k-1) !}{(2 \pi i)^{2}} \oint_{\Gamma_{\tau, \varepsilon}} \frac{d \zeta}{(\zeta-\tau)^{k}} \oint_{|z|=r_{0}} \frac{f(\zeta, z)}{z^{2(n-k)+1}} d z
$$

where $r_{0}<r / 2$ is fixed, but arbitrary.

By the restriction of $\tau$ in $S\left(0, \alpha^{\prime} ; T / 2\right)$ and the definition of $\Gamma_{\tau, \varepsilon}$, there is a positive constant $c>0$ depending only on $\varepsilon$ such that

$$
|\tau| / 2 \leq|\zeta| \leq 2|\tau| \quad \text { and } \quad c|\tau| \leq|\zeta-\tau| \quad \text { for all } \zeta \in \Gamma_{\tau, \varepsilon}
$$

Therefore, from the estimate (2.3) we got

$$
\left|\frac{d^{k-1}}{d \tau^{k-1}} f_{2(n-k)}(\tau)\right| \leq \frac{C}{r_{0}^{2 n}} \frac{r_{0}^{2 n}}{c^{k}}\{2(n-k)\} !(k-1) ! \frac{e^{-\delta /|\tau|}}{|\tau|^{k-1}},
$$

for some positive constants $C$ and $\delta$. By using $\{2(n-k) !\} !(k-1) ! \leq$ $(2 n) ! /(k-1) !,(2.5)$ yields

$$
\left|w_{+}(\tau, z)\right| \leq C \frac{r_{0}^{2}}{c} \sum_{n=1}^{\infty} \frac{|z|^{2 n}}{r_{0}^{2 n}} \exp \left(-\left(\delta-r_{0}^{2} / c\right) /|\tau|\right) .
$$

Therefore, if $r_{0}$ is sufficiently small and $|z|<r_{0}$, the sum converges and $w_{+}$ is exponentially small. This completes the proof.

To end the proof of Theorem 2.1, we remark that the existence of infinitely many actual solutions is shown as follows. For the formal solution $\hat{u}(\tau, z)$ we know that there are infinitely many $u(\tau, z)$ such that $u(\tau, z) \sim_{1}$ $\hat{u}(\tau, z)$ in $S(\theta, \pi)$ by the expression (1.10). Also the arbitrariness of $\psi_{i}(\tau)$ $(i=1,2)$ in the Cauchy problem (2.1) shows the existence of infinitely many actual solutions. In order to see such solutions directly, the following example is useful.

EXAMPLE 2.1. Let

$$
u_{n}(\tau, z)=\frac{1}{\sqrt{\tau}} \exp \left(-\frac{\left(z-n e^{i \theta / 2}\right)^{2}}{4 \tau}\right), \quad|z| \leq 1
$$

be a sequence of analytic solutions of the heat equation. For a given sector $S(\theta, \alpha)(\alpha<\pi)$, we choose $N$ such that $\operatorname{Re}\left\{\left(z-N e^{i \theta / 2}\right)^{2} / 4 \tau\right\}>0$ for $|z| \leq 1, \tau \in S(\theta, \alpha)$. Then $u_{n}(\tau, z) \sim_{1} 0$ in $S(\theta, \alpha)$ for any $n \geq N$. 


\section{$\S$ 3. Fine Borel summability - Proof of main results}

We first remark that if the formal solution $\hat{u}(\tau, z)$ is finely Borel summable in a direction $\theta$, then its fine Borel $\operatorname{sum} u^{\theta}(\tau, z)$ is an actual analytic solution of the heat equation. Indeed,

$$
\frac{\partial}{\partial \tau} u^{\theta}(\tau, z)-\frac{\partial^{2}}{\partial z^{2}} u^{\theta}(\tau, z) \sim_{1} 0, \quad \text { finely in the direction } \theta
$$

implies the assertion (cf. $\S 1.7$ ).

Now our main result (B) is an immediate consequence of the following theorem in view of Theorem 1.3.

Main Theorem 3.1. Let $v(s, z)$ be the formal Borel transform of the formal solution $\hat{u}(\tau, z)$ given by (0.2). Then the following two statements are equivalent:

(i) $v(s, z)$ is analytic on $E_{+}(\theta, w) \times B(r)$ for some positive constants $w$ and $r$, and satisfies the exponential type growth condition as $s \rightarrow \infty$ in $E_{+}(\theta ; w)$. Here

$$
E_{+}(\theta, w)=\left\{s \in \mathbb{C} \mid \operatorname{dist}\left(s, e^{i \theta} \mathbb{R}_{+}\right)<w\right\} .
$$

(ii) The Cauchy data $\varphi(z)$ is analytic on $\Omega(\theta / 2, \omega)$ for some positive constant $\omega$, and satisfies the growth condition of exponential order at most 2 as $z \rightarrow \infty$ in $\Omega(\theta / 2, \omega)$. Here

$$
\Omega(\theta / 2, \omega)=\left\{z \in \mathbb{C} \mid \operatorname{dist}\left(z, e^{i \theta} \mathbb{R}\right)<\omega\right\} .
$$

Remark 3.1. The proof below shows that $v(s, z)$ is actually analytic on $E_{+}\left(\theta, w^{\prime}\right) \times \Omega\left(\theta / 2, \omega^{\prime}\right)$ for small $w^{\prime}$ and $\omega^{\prime}$, and satisfies the growth condition

$$
|v(s, z)| \leq C e^{A|s|+B|z|^{2}} \quad \text { on } E_{+}\left(\theta, w^{\prime}\right) \times \Omega\left(\theta / 2, \omega^{\prime}\right)
$$

for some positive constants $A, B$ and $C$. This implies that the Borel sum $u^{\theta}(\tau, z)$ in the direction $\theta$, which is given by (1.12), is analytic on $O(\theta, T) \times$ $\Omega\left(\theta / 2, \omega^{\prime \prime}\right)$ for some positive constants $T$ and $\omega^{\prime \prime}$. More precise properties will be given after the proof of this theorem.

Proof of (ii) $\Rightarrow$ (i). Suppose that the Cauchy data $\varphi(z)$ satisfies the conditions in (ii). We take a positive constant $r$ so that $B(2 r) \subset \Omega(\theta / 2, \omega)$. 
Then, by the definition of the formal Borel transform, we have for $z \in B(r)$

$$
\begin{aligned}
v(s, z) & =\sum_{n=0}^{\infty} \varphi^{(2 n)}(z) \frac{s^{n}}{(n !)^{2}} \\
& =\sum_{n=0}^{\infty} \frac{s^{n}}{(n !)^{2}} \frac{(2 n) !}{2 \pi i} \oint_{|\zeta|=r} \frac{\varphi(\zeta+z)}{\zeta^{2 n+1}} d \zeta \\
& =\frac{1}{2 \pi i} \oint_{|\zeta|=r} \sum_{n=0}^{\infty} \frac{(2 n) !}{(n !)^{2}} \frac{s^{n}}{\zeta^{2 n+1}} \varphi(\zeta+z) d \zeta
\end{aligned}
$$

Since $\sum_{n=0}^{\infty} \xi^{n}(2 n) ! /(n !)^{2}=1 / \sqrt{1-4 \xi}(|\xi|<1 / 4)$, we have

$$
\frac{1}{\zeta} \sum_{n=0}^{\infty} \frac{(2 n) !}{(n !)^{2}} \frac{s^{n}}{\zeta^{2 n}}=\frac{1}{\sqrt{\zeta^{2}-4 s}}, \quad 4|s|<|\zeta|^{2},
$$

where $\sqrt{\zeta^{2}}:=\zeta$ for $s=0$. Hence we have

$$
v(s, z)=\frac{1}{2 \pi i} \oint_{|\zeta|=r} \frac{\varphi(\zeta+z)}{\sqrt{\zeta^{2}-4 s}} d \zeta, \quad|s|<r^{2} / 4,|z|<r .
$$

Since $\sqrt{\zeta^{2}-4 s}(s \neq 0)$ is univalent in $\zeta$-plane outside a segment joining two points $\{ \pm 2 \sqrt{s}\}$, we can deform the contour of integration into any simple, piecewise smooth curve $\Gamma$ surrounding this segment such that $z+\Gamma$ is contained in $\Omega(\theta / 2, \omega)$, the domain of holomorphy of $\varphi(\zeta)$.

We take a small positive constant $w$ such that $E_{+}(\theta, w) \subset\left\{z^{2} / 4 \mid z \in\right.$ $\Omega(\theta / 2, \omega / 3)\}$. By the definition, $\pm 2 \sqrt{s} \in \Omega(\theta / 2, \omega / 3)$ for any $s \in E_{+}(\theta, w)$. Therefore $\mathrm{w}$ can deform the contour in (3.1) to a rectangle $\Gamma$ consisting of the boundary of $\Omega(\theta / 2, \omega / 2)$ by cutting it off at $\left|\operatorname{Re}\left(\zeta e^{-i \theta / 2}\right)\right|=$ $2|s|^{1 / 2}+1$. By this choice of $\Gamma$, we see that $v(s, z)$ can be contined analytically on $E_{+}(\theta, w) \times \Omega(\theta / 2, \omega / 3)$, because $z+\Gamma \subset \Omega(\theta / 2,5 \omega / 6)$ for any $z \in \Omega(\theta / 2, \omega / 3)$.

Here we recall the growth condition for $\varphi(z)$ that $|\varphi(z)| \leq C \exp \left(\delta|z|^{2}\right)$, $(z \in \Omega(\theta / 2, \omega))$ for some positive constants $C$ and $\delta$. Then the above choice of the contour $\Gamma$ implies the following growth estimate for $v(s, z)$,

$$
|v(s, z)| \leq C^{\prime} e^{A|s|+B|z|^{2}}, \quad(s, z) \in E_{+}(\theta, w) \times \Omega(\theta / 2, \omega / 3) .
$$

for some positive constants $C^{\prime}, A$ and $B$. In fact, it is sufficient to notice the following inequalities for $\zeta \in \Gamma$ and $s \in E_{+}(\theta, w)$,

$$
|z+\zeta| \leq|z|+2|s|^{1 / 2}+1, \quad\left|\zeta^{2}-4 s\right| \geq \omega / 6
$$


Proof of (i) $\Rightarrow$ (ii). The formal Borel transform $v(s, z)$ of the formal solution $\hat{u}(\tau, z)$ is assumed to be analytic on $E_{+}(\theta, w) \times B(r)$, and $\max _{|z| \leq r}$ $|v(s, z)|$ is assumed to satisfy the growth condition of exponential type as $s \rightarrow \infty$ in $E_{+}(\theta, w)$.

First, we remark that $v(s, z)$ satisfies the following Fuchs type partial differential equation,

$$
\left(\frac{\partial}{\partial s} s \frac{\partial}{\partial s}\right) v(s, z)=\frac{\partial^{2}}{\partial z^{2}} v(s, z) .
$$

In fact, this is deduced from the following commutative diagram;

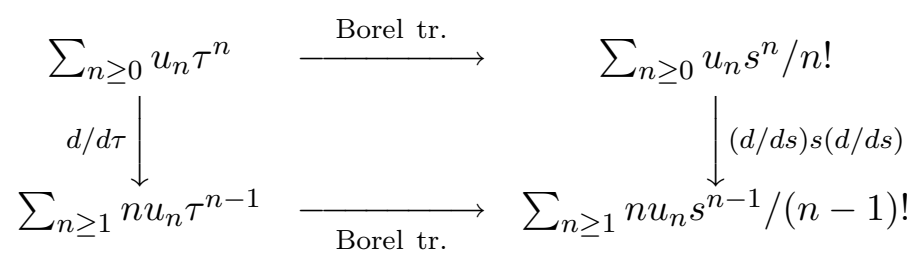

Now we regard $v(s, z)$ as a solution of the Cauchy problem to the equation (3.3) with data $v\left(s, z_{0}\right)$ and $v_{z}\left(s, z_{0}\right)$ at $z=z_{0}$.

$$
v\left(s, z_{0}\right)=\psi_{0}(s), \quad \frac{\partial v}{\partial z}\left(s, z_{0}\right)=\psi_{1}(s) .
$$

For simplicity, we put Then by the assumption on $v(s, z), \psi_{i}(s)(i=0,1)$ are analytic on $E_{+}(\theta, w)$, and satisfy the exponential type growth condition on the domain.

Here we introduce a bell type domain $\Delta(\theta / 2, w)$ in $z$-plane, defined by

$$
\Delta(\theta / 2, w):=\left\{z \in \mathbb{C} \mid z^{2} / 4 \in E_{+}(\theta, w)\right\}
$$

which contains the line $e^{i \theta / 2} \mathbb{R}$ and shrinks to this line as $z \rightarrow \infty$.
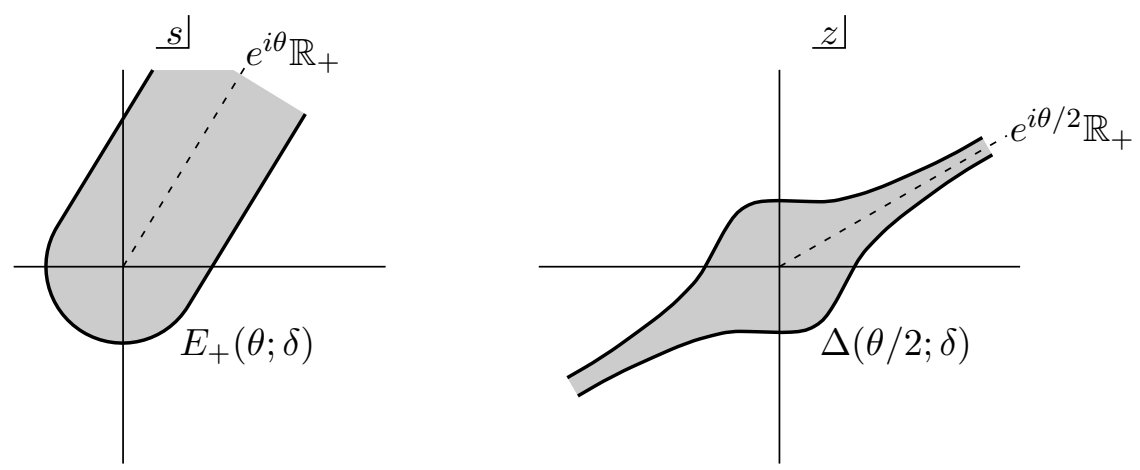
We shall prove that if we take small $r^{\prime}$, then for the solution $v(s, z)$ of the Cauchy problem (3.3)-(3.5) with $z_{0} \in B\left(r^{\prime}\right), v(0, z)(=\varphi(z))$ can be continued analytically on $z_{0}+\Delta(\theta / 2, w / 2)$, and satisfies the growth condition of exponential order at most 2 on the domain. If this will be proved, the assertion (i) follows from the fact that $\bigcup_{z_{0} \in B\left(R^{\prime}\right)}\left\{z_{0}+\Delta(\theta / 2, w / 2)\right\}$ contains $\Omega(\theta / 2, \omega)$ for small $\omega>0$.

To prove the above assertion, we may assume $\psi_{0}(s) \equiv 0$ without loss of generality, and we write $\psi_{1}(s)$ by $\psi(s)$. Then the solution $v(s, z)$ of the Cauchy problem (3.3)-(3.5) has the following expansion in a neighborhood of $z_{0}$,

$$
v(s, z)=\sum_{n=0}^{\infty}\left(\frac{\partial}{\partial s} s \frac{\partial}{\partial s}\right)^{n} \psi(s) \frac{\left(z-z_{0}\right)^{2 n+1}}{(2 n+1) !} .
$$

Since

$$
\left(\frac{\partial}{\partial s} s \frac{\partial}{\partial s}\right)^{n} \psi(0)=n ! \psi^{(n)}(0)
$$

We get the following integral expression of $v(0, z)$ in a neighborhood of $z_{0}$.

$$
\begin{aligned}
v(0, z) & =\sum_{n=0}^{\infty} \psi^{(n)}(0) \frac{n !}{(2 n+1) !}\left(z-z_{0}\right)^{2 n+1} \\
& =\frac{1}{2 \pi i} \sum_{n=0}^{\infty} \oint_{|\sigma|=w} \frac{n ! n !}{(2 n+1) !} \frac{\left(z-z_{0}\right)^{2 n+1}}{\sigma^{n+1}} \psi(\sigma) d \sigma \\
& =\frac{z-z_{0}}{2 \pi i} \oint_{|\sigma|=w}{ }_{2} F_{1}\left(1,1 ; \frac{3}{2} ; \frac{\left(z-z_{0}\right)^{2}}{4 \sigma}\right) \frac{\psi(\sigma)}{\sigma} d \sigma \\
& \left(\left|z-z_{0}\right|^{2} / 4<w\right)
\end{aligned}
$$

where ${ }_{2} F_{1}$ is the Gauss hypergeometric function which is given by

$$
{ }_{2} F_{1}(\alpha, \beta ; \gamma ; \zeta)=\sum_{n=0}^{\infty} \frac{(\alpha)_{n}(\beta)_{n}}{(\gamma)_{n}} \frac{\zeta^{n}}{n !}, \quad \text { for }|\zeta|<1
$$

where $(\alpha)_{n}:=\alpha(\alpha+1) \cdots(\alpha+n-1)(n \geq 1)$ and $(\alpha)_{0}:=1$ for $\alpha \in \mathbb{C}$ and continued analytically to $\mathbb{C} \backslash[1, \infty)$.

Since ${ }_{2} F_{1}(1,1 ; 3 / 2 ; \zeta)$ has possible singular points $\{1, \infty\}$ of regular singular type, it therefore satisfies a growth condition of polynomial type as $\zeta \rightarrow 1$ and $\zeta \rightarrow \infty$ in any sector with finite opening angle. Now we restrict 
$z_{0}$ in $\left|z_{0}\right|<2 \sqrt{w}$ so that $v(0, z)$ is defined at $z=0$ in the expression (3.7). By the definition of the domain $\Delta(\theta / 2, w / 2)$, we have

$$
\inf \left\{\operatorname{dist}\left(\left(z-z_{0}\right)^{2} / 4, \partial E_{+}(\theta, w)\right) \mid z \in z_{0}+\Delta(\theta / 2, w / 2)\right\}=w / 2 .
$$

From the expression (3.7), we already know that $v(0, z)$ is defined for $z$ such that $\left|z-z_{0}\right|^{2} / 4 \leq w / 2$, and such points $z$ belong to $z_{0}+\Delta(\theta / 2, w / 2)$.

In the integral expression (3.7), for $z \in z_{0}+\Delta(\theta / 2, w / 2)$ we deform the contour to a simple closed curve $\Gamma$ consisting of $\partial E_{+}(\theta, w)$ by cutting at $\operatorname{Re}\left(\sigma e^{-i \theta}\right)=|z|^{2}+1$. Then we see that $v(0, z)$ can be continued to $z=\infty$ in $z_{0}+\Delta(\theta / 2, w / 2)$. Finally, we can easily see that $v(0, z)$ has the growth condition of exponential order at most 2 in the mentioned domain, from the new integral expression for $v(0, z)$ and the growth condition for $\psi(s)$.

This completes the proof.

As a corollary to the Main Theorem, we can characterize Cauchy data with Borel summable formal solutions in sectors of opening angle larger than $\pi$ as follows.

THEOREM 3.2. The formal solution $\hat{u}(\tau, z)$ is Borel summable in a sector $S(\theta, \alpha)$ with $\alpha>\pi$ if and only if the Cauchy data $\varphi(z)$ can be continued analytically in a domain consisting a two sided infinite sector $D(\theta, \alpha):=S(\theta / 2,(\alpha-\pi) / 2 ; \infty) \cup S(\pi+(\theta / 2),(\alpha-\pi) / 2 ; \infty)$ and satisfies the growth condition of exponential order at most 2 as $z \rightarrow \infty$ in $D\left(\theta, \alpha^{\prime}\right)$ for any $\alpha^{\prime}<\alpha$, i.e.,

$$
|\varphi(z)| \leq C e^{\delta|z|^{2}}, \quad x \in D\left(\theta, \alpha^{\prime}\right),
$$

where $C$ and $\delta$ may depend upon $\alpha^{\prime}$.

Hence the formal solution $\hat{u}(\tau, z)$ is Borel summable in a sector with an opening angle larger than $3 \pi$ if and only if $\varphi(z)$ is an entire function of exponential order at most 2, which is nothing but the condition of Kowalevskaya's result stated in Introduction.

Proof. First notice that if the formal solution $\hat{u}(\tau, z)$ is Borel summable in $S(\theta, \alpha)$, where $\alpha>\pi$, then it is finely Borel summable in each direction $\eta$ with $|\eta-\theta|<\left(\alpha^{\prime}-\pi\right) / 2$ for any $\alpha^{\prime}<\alpha$. This proves the "if" part. Next, if the Cauchy data $\varphi(z)$ satisfies the above condition then it is easily seen that the formal Borel transform $v(s, z)$ satisfies the growth condition as $s \rightarrow \infty$ in $S\left(\theta, \alpha^{\prime}-\pi\right)$ for any $\alpha^{\prime}<\alpha$ as in the proof of theorem 3.1. Hence by 
theorem 1.2, the formal solution is Borel summable in $S(\theta, \alpha)$. This proves the theorem.

ExAMPLE 3.1. (Example of a just finely summable case) Let $\varphi(z)=$ $e^{i z^{3}}$ or $\sin z^{3}$. Then it is easily verified that $\varphi(z)$ satisfies the growth condition of exponential order 2 in $\Omega(0, \omega)$ for any fixed $\omega>0$, but the same growth condition never holds in any sector $S(0, \alpha ; \infty),(\alpha>0)$. By taking this $\varphi(z)$ as a Cauchy data, we have an example of formal solution $\hat{u}(\tau, z)$ which is finely Borel summable in 0-direction but is not Borel summable in a sectorial domain of opening angle larger than $\pi$.

Next we shall give an improvement of Gevrey asymptotic estimates and an integral expression of the fine Borel sum.

THEOREM 3.3. Let suppose the formal solution $\hat{u}(\tau, z)=\sum_{n=0}^{\infty} u_{n}(z) \tau^{n}$ to be finely Borel summable in a direction $\theta$ with Cauchy data $u_{0}(z)=\varphi(z)$, and $u^{\theta}(\tau, z)$ be its fine sum defined on $O(\theta, T) \times \Omega(\theta / 2, \omega)$. Then we have:

(i) (Gevrey asymptotic estimates) For some $T^{\prime}<T$, $\omega^{\prime}<\omega$, there exist positive constants $C, K$ and $\delta$ such that the following asymptotic estimates hold.

$$
\begin{array}{r}
\left|u^{\theta}(\tau, z)-\sum_{n=0}^{N-1} u_{n}(z) \tau^{n}\right| \leq C K^{N} N ! e^{\delta|z|^{2}}|\tau|^{N}, \\
(\tau, z) \in O\left(\theta, T^{\prime}\right) \times \Omega\left(\theta / 2, \omega^{\prime}\right)
\end{array}
$$

for all $N=1,2,3, \ldots$.

(ii) (integral expression) The fine Borel sum $u^{\theta}(\tau, z)$ has the following integral expression involving the heat kernel, $e^{-\zeta^{2} /(4 \tau)} / \sqrt{4 \pi \tau}$.

$$
u^{\theta}(\tau, z)=\frac{1}{\sqrt{4 \pi \tau}} \int_{-\infty e^{i \theta / 2}}^{\infty e^{i \theta / 2}} e^{-\zeta^{2} /(4 \tau)} \varphi(z+\zeta) d \zeta .
$$

Proof of (i). By Theorem 3.1, the Cauchy data $\varphi(z)$ is analytic on $\Omega(\theta / 2, w)$ for some positive constant $w$, and satisfies there a growth condition of exponential order at most 2. We recall that the fine Borel sum $u^{\theta}(\tau, z)$ has an integral expression (cf. (1.12)),

$$
u^{\theta}(\tau, z)=\frac{1}{\tau} \int_{0}^{\infty e^{i \theta}} e^{-s / \tau} v(s, z) d s
$$


where $v(s, z)$ is the formal Borel transform of $\hat{u}(\tau, z)$, and satisfies (from (3.2)) the following inequality

$$
|v(s, z)| \leq C e^{A|s|+B|z|^{2}}, \quad(s, z) \in E_{+}\left(\theta, w^{\prime}\right) \times \Omega\left(\theta / 2, \omega^{\prime}\right)
$$

for some positive constants $A, B, C, w^{\prime}$ and $\omega^{\prime}$.

$$
\begin{aligned}
& \left|\frac{\partial^{n} v}{\partial s^{n}}(s, z)\right| \leq C^{\prime} K^{n} n ! e^{A|s|+B|z|^{2}}, \\
& \quad(s, z) \in E_{+}\left(\theta, w^{\prime \prime}\right) \times \Omega\left(\theta / 2, \omega^{\prime}\right)
\end{aligned}
$$

Hence by Cauchy's integral formula for any $w^{\prime \prime}<w^{\prime}$ for suitable positive constants $C^{\prime}$ and $K$.

Now by the repeated use of integration by parts in (3.10), we have

$$
u^{\theta}(\tau, z)-\sum_{n=0}^{N-1} u_{n}(z) \tau^{n}=u_{N}(z) \tau^{N}+\tau^{N} \int_{o}^{\infty e^{i \theta}} e^{-s / \tau} \frac{\partial^{N+1}}{\partial s^{N+1}} v(s, z) d s,
$$

for $\tau$ such that $\operatorname{Re}\left(e^{i \theta} / \tau\right)>2 A$, and $z \in \Omega\left(\theta / 2, \omega^{\prime}\right)$. Here, $u_{n}(z)=\left(\partial^{n} v / \partial s^{n}\right)(0, z)$.

By restricting $\tau$ as above, the integral part in the above equality is estimated by

$$
\begin{aligned}
C^{\prime}|\tau|^{N} K^{N}(N+1) ! e^{B|z|^{2}} \int_{0}^{\infty} e^{-A r} d r & =C^{\prime} A^{-1}|\tau|^{N} K^{N}(N+1) ! e^{B|z|^{2}} \\
& \leq C^{\prime \prime} K^{\prime N} N ! e^{B|z|^{2}}|\tau|^{N}
\end{aligned}
$$

for some positive constants $C^{\prime \prime}$ and $K^{\prime}$. Here we notice that $\left\{\tau \mid \operatorname{Re}\left(e^{i \theta} / \tau\right)>\right.$ $2 A\}=\left\{\tau|| \tau-e^{i \theta} / A \mid<1 / A\right\}=O(\theta, 1 / A)$. Combining the results above, we have the desired asymptotic estimates (3.8).

Proof of (ii). First we recall the integral expression of $v(s, z)$ given by $(3.1)$,

$$
v(s, z)=\frac{1}{2 \pi i} \int_{\Gamma} \frac{\varphi(z+\zeta)}{\sqrt{\zeta^{2}-4 s}} d \zeta,
$$

where $\Gamma$ is any simple closed piecewise smooth curve surrounding the segment joining two points $\{ \pm 2 \sqrt{s}\}(s \neq 0)$. We restrict $s$ to the ray $\left\{r e^{i \theta} \mid\right.$ $r \geq 0\}$, and put $\sqrt{s}:=\sqrt{r} e^{i \theta / 2}$.

Let $r>0$ and $\zeta=\xi+i \eta$, where $|\xi|<2 \sqrt{r}$. Then by noticing

$$
\sqrt{(\xi+i 0)^{2}-4 r}=i \sqrt{4 r-\xi^{2}}, \quad \sqrt{(\xi-i 0)^{2}-4 r}=-i \sqrt{4 r-\xi^{2}},
$$


we have

$$
v(s, z)=v\left(r e^{i \theta}, z\right)=\frac{1}{\pi} \int_{-2 \sqrt{r}}^{2 \sqrt{r}} \frac{\varphi\left(z+\xi e^{i \theta / 2}\right)}{\sqrt{4 r-\xi^{2}}} d \xi, \quad r>0 .
$$

Then by substituting this into (3.10), we have

$$
\begin{aligned}
u^{\theta}(\tau, z) & =\frac{e^{i \theta}}{\pi \tau} \int_{0}^{\infty} \exp \left(-\frac{e^{i \theta}}{\tau} r\right) d r \int_{-2 \sqrt{r}}^{2 \sqrt{2}} \frac{\varphi\left(z+\xi e^{i \theta / 2}\right)}{\sqrt{4 r-\xi^{2}}} d \xi \\
& =\frac{e^{i \theta / 2}}{\pi \tau} \int_{-\infty}^{\infty} \varphi\left(z+\xi e^{i \theta / 2}\right) d\left(\xi e^{i \theta / 2}\right) \int_{\xi^{2} / 4}^{\infty} \exp \left(-\frac{e^{i \theta}}{\tau} r\right) \frac{d r}{\sqrt{4 r-\xi^{2}}}
\end{aligned}
$$

Now the desired integral expression (3.9) is a consequence of the

$$
\begin{aligned}
& \int_{\xi^{2} / 4}^{\infty} \exp \left(-\frac{e^{i \theta}}{\tau} r\right) \frac{d r}{\sqrt{4 r-\xi^{2}}} \quad\left(x=\sqrt{4 r-\xi^{2}}\right) \\
& \quad=\frac{1}{2} \exp \left(-\frac{\left(\xi e^{i \theta / 2}\right)^{2}}{4 \tau}\right) \int_{0}^{\infty} \exp \left(-\frac{e^{i \theta}}{4 \tau} x^{2}\right) d x \quad\left(y=x e^{i \theta / 2} /(2 \sqrt{\tau})\right) \\
& =e^{-i \theta / 2} \frac{\sqrt{\pi \tau}}{2} \exp \left(-\frac{\left(\xi e^{i \theta / 2}\right)^{2}}{4 \tau}\right)
\end{aligned}
$$

following calculations. This completes the proof.

\section{$\S 4$. Factorial series expansion}

In this section, we shall give a factorial series expansion for the Borel sum $u^{\theta}(\tau, z)$ of $\hat{u}(\tau, z)$ in the $\theta$ direction,

$$
u^{\theta}(\tau, z)=\frac{1}{\tau} \int_{0}^{\infty(\theta)} e^{-s / \tau} v(s, z) d s
$$

where $v(s, z)$ is the formal Borel transform of $\hat{u}(\tau, z)$ and is assumed to be analytic on $E_{+}(\theta, w) \times B(r)$ and satisfy the growth condition,

$$
\max _{z \in B(r)}|v(s, z)| \leq C e^{A|s|}, \quad s \in E_{+}(\theta, w) .
$$

Although we restrict the variable $z$ in $B(r)$ for the simplicity, the results will hold for $z \in \Omega(\theta / 2, \omega)$ by using the asymptotic estimate (3.8).

First we give a formal procedure to get the factorial series expansion of $u^{\theta}(\tau, z)$ (cf. [Was, Chap. XI]). We choose and fix a positive constant $\kappa$ 
such that $\kappa<2 w / \pi$ or $w / \kappa>2 \pi$. We transform a variable $s$ to $p$ by $p=$ $s e^{-i \theta} / \kappa$. Then by this change of variables, the domain $E_{+}(\theta, w)$ corresponds to $E_{+}(0, \rho)$ with $\rho=w / \kappa>\pi / 2$. We put $g(p, z)=v\left(e^{i \theta} \kappa p, z\right)$. Then we have

$$
\max _{z \in B(r)}|g(p, z)| \leq C e^{A \kappa|p|}, \quad p \in E_{+}(0, \rho)(\rho>\pi / 2)
$$

We further change the variable $p$ to $\eta$ by $\eta=e^{-p}$, and put $f(\eta, z)=$ $f(\log (1 / \eta), z)=v\left(e^{i \theta} \kappa \log (1 / \eta), z\right)$. By this change of variables the domain $E_{+}(0, \rho)$ corresponds to a domain $D$ containing $O(0, h):=\{\eta \in \mathbb{C}|| \eta-h \mid<$ $h\}\left({ }^{\exists} h>1\right)$.
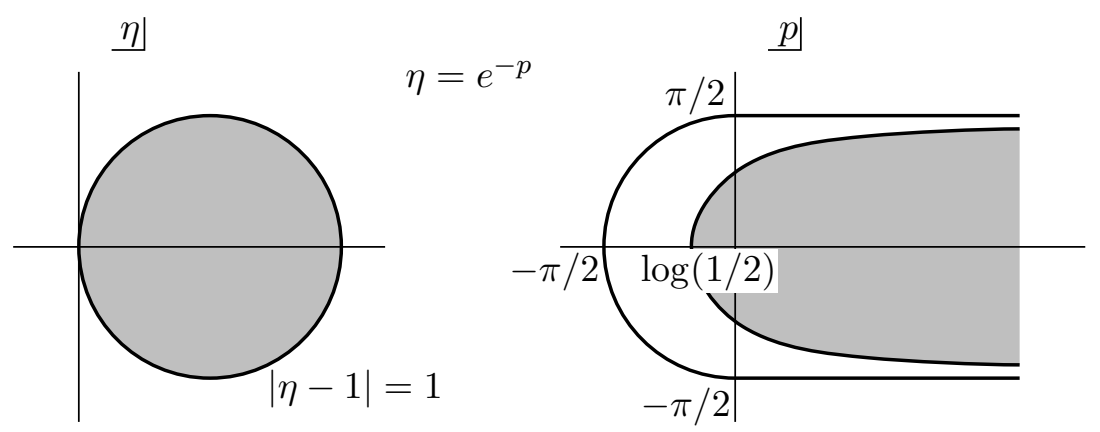

By definition, $f(\eta, z)(z \in B(r))$ is analytic on $\{|\eta-h| \leq h\} \backslash\{0\}$, and satisfies the following growth condition as $\eta \rightarrow 0$,

$$
\max _{z \in B(r)}|f(\eta, z)| \leq C|\eta|^{-A \kappa}, \quad|\eta-h|<h
$$

By the above changes of variables, we see that $u^{\theta}(\tau, z)$ is given by

$$
u^{\theta}(\tau, z)=\frac{e^{i \theta} \kappa}{\tau} \int_{0}^{1} \eta^{\left(e^{i \theta} \kappa / \tau\right)-1} f(\eta, z) d \eta
$$

Since $f(\eta, z)$ is analytic on $O(0,1) \times B(r)(O(0,1) \subset O(0, h))$, it has a Taylor expansion

$$
f(\eta, z)=\sum_{n=0}^{\infty} \phi_{n}(z)(1-\eta)^{n}, \quad(\eta, z) \in O(0,1) \times B(r)
$$


Now by substituting this expansion into (4.5), a formal calculation leads to the following factorial series expansion

$$
\begin{aligned}
u^{\theta}(\tau, z) & =\sum_{n=0}^{\infty} \phi_{n}(z) \frac{n !}{\left(\left(e^{i \theta} \kappa / \tau\right)+1\right) \cdots\left(\left(e^{i \theta} \kappa / \tau\right)+n\right)} \\
& =\sum_{n=0}^{\infty} \phi_{n}(z) \frac{n ! \tau^{n}}{\left(e^{i \theta} \kappa+\tau\right) \cdots\left(e^{i \theta} \kappa+n \tau\right)}, \quad \operatorname{Re}\left(e^{i \theta} / \tau\right)>0 .
\end{aligned}
$$

where, here and in the sequel, the term corresponding to $n=0$ in the sums is meant to be $\varphi(z)$. Now we shall prove the following.

THEOREM 4.1. Assume that $\varphi(z)$ satisfies the conditions in theorem 3.1 (ii). Then choosing $\kappa$ as above, the Borel sum (4.1) can be represented as (4.7) which converges absolutely and uniformly on $O(\theta, 1 /(2 B)) \times B(r)$ for every $B>A$, where $O(\theta, 1 /(2 B))=\left\{\mid r-e^{i \theta} /(2 B)<1 /(2 B)\right\}=$ $\left\{\tau \mid \operatorname{Re}\left(e^{i \theta} / \tau\right)>B\right\}$. Moreover the following estimates hold for all $N=$ $1,2,3, \ldots$,

$$
\left|u^{\theta}(\tau, z)-\sum_{n=0}^{N-1} \phi_{n}(z) \frac{n ! \tau^{n}}{\left(e^{i \theta} \kappa+\tau\right) \cdots\left(e^{i \theta} \kappa+n \tau\right)}\right| \leq \frac{C}{N^{(B-A) \kappa}}
$$

$((\tau, z) \in O(\theta, 1 /(2 B)) \times B(r))$, for some positive constant $C$ only depending on $B>A$.

For the proof, the following lemma plays a crucial role.

Lemma 4.2. Let $v(s, z)$ satisfy the inequality (4.2). Then the Taylor coefficients $\left\{\phi_{n}(z)\right\}$ of $f(\eta, z)$ satisfy

$$
\max _{z \in B(r)}\left|\phi_{n}(z)\right| \leq C n^{A \kappa-1}
$$

for some positive constant $C$.

For the case where $A \kappa>1$, one can find a proof in G. Doetsch [Doe, Chap. 11, p. 207]. For the case $0<A \kappa \leq 1$, recall the relation $f(\eta, z)=$ $v\left(e^{i \theta} \kappa \log (1 / \eta), z\right)$. This shows

$$
\frac{\partial f}{\partial \eta}(\eta, z)=-\frac{e^{i \theta} \kappa}{\eta} \frac{\partial v}{\partial s}(s, z), \quad s=e^{i \theta} \kappa \log (1 / \eta)
$$


Since $v(s, z)$ satisfies the growth condition (4.2), we may assume that $\partial v(s, z) / \partial s$ also satisfies the same growth condition by Cauchy's integral formula. Hence we have

$$
\max _{z \in B(r)}\left|\frac{\partial f}{\partial \eta}(\eta, z)\right| \leq C^{\prime}|\eta|^{-A \kappa-1}, \quad|\eta-1|<1 .
$$

Hence the first case shows that $n\left|\phi_{n}(z)\right| \leq C^{\prime \prime} n^{A \kappa}(z \in B(r))$, which implies the desired estimates.

Proof of Theorem 4.1. Let $B>A$ and we restrict $\tau$ such that $\operatorname{Re}\left(e^{i \theta} / \tau\right)$ $\geq B$, i.e., $\tau \in O(\theta, 1 /(2 B))$. Then by the above lemmas we have

$$
\begin{aligned}
& \max _{z \in B(r)}\left|\phi_{n}(z)\right|\left|\frac{n ! \tau^{n}}{\left(e^{i \theta} \kappa+\tau\right) \cdots\left(e^{i \theta} \kappa+n \tau\right)}\right| \\
& \leq C n^{A \kappa-1} \frac{n !}{(B \kappa+1) \cdots(B \kappa+n)} \leq C^{\prime} \frac{\Gamma(B \kappa+1)}{n^{(B-A) \kappa+1}} .
\end{aligned}
$$

This shows that the factorial series (4.7) converges absolutely and uniformly on $O(\theta, 1 /(2 B)) \times B(r)$ and the remainder estimate (4.8) follows. On the other hand, for $0<\eta \leq 1$ we have, by Lemma 4.2 ,

$$
\begin{aligned}
& \sum_{n=0}^{\infty}\left|\phi_{n}(z)\right|(1-\eta)^{n} \leq C \sum_{n=0}^{\infty} n^{A \kappa-1}(1-\eta)^{n} \\
& \quad \leq C^{\prime} \sum_{n=0}^{\infty} \frac{A \kappa(A \kappa+1) \cdots(A \kappa+n-1)}{n !}(1-\eta)^{n}=C^{\prime} \eta^{-A \kappa} .
\end{aligned}
$$

Here, the last equality follows from $(1-y)^{-\alpha}=\sum_{n=0}^{\infty}\{\alpha(\alpha+1) \cdots(\alpha+$ $n-1) / n !\} y^{n}(|y|<1)$. Hence we have for $(\tau, z) \in O(\theta, 1 /(2 B)) \times B(r)$,

$$
\left|\eta^{\left(e^{i \theta} \kappa / \tau\right)-1}\right| \sum_{n=0}^{\infty}\left|\phi_{n}(z)\right|(1-\eta)^{n} \leq C \eta^{(B-A) \kappa-1}, \quad 0<\eta \leq 1,
$$

which is integrable on $(0,1]$. This permits the change of order of integration and summation in (4.5), which proves the theorem.

From the well known relation between formal power series and factorial series (cf. Wasow [Was, section 46.3], it follows that the coefficients $\left\{\phi_{n}(z)\right\}_{n=0}^{\infty}$ in the factorial series expansion (4.7) are linear combinations of $\left\{\varphi(z), \varphi^{(2)}(z), \ldots, \varphi^{(2 n)}(z)\right\}$ with constant coefficients. Below, we shall give a recurrence formula to determine them directly from the data. 
THEOREM 4.3. We have the following recurrence formula for $\left\{\phi_{n}(z)\right\}_{n=0}^{\infty}\left(\right.$ here $\left.\lambda=\kappa e^{i \theta}\right)$ :

$$
\begin{gathered}
\phi_{0}(z)=\varphi(z), \quad \phi_{1}(z)=\lambda \varphi^{\prime \prime}(z) \\
\phi_{2}(z)=\frac{\lambda}{2} \varphi^{(2)}(z)+\frac{\lambda^{2}}{4} \varphi^{(4)}(z) . \\
(n+1)^{2} \phi_{n+1}(z)=\lambda \phi_{n}^{\prime \prime}(z)+\frac{(3 n+1) n}{2} \phi_{n}(z) \\
-\sum_{j=1}^{n} \frac{(2 n-j)(n-j)}{j(j+1)(j+2)} \phi_{n-j}(z), \quad n \geq 2 .
\end{gathered}
$$

Proof. We will first derive a factorial series expansion of $\partial u(\tau, z) / \partial \tau$ and then compare it to that of $\partial^{2} u(\tau, z) / \partial z^{2}$.

For that purpose, put

$$
f_{n}(\tau, \lambda)=\frac{n ! \tau^{n}}{(\lambda+\tau)(\lambda+2 \tau) \cdots(\lambda+n \tau)}
$$

and calculate $d f_{n}(\tau, \lambda) / d \tau$.

$$
\begin{aligned}
\frac{d}{d \tau} f_{n}(\tau, \lambda)= & \frac{d}{d \tau}\left\{\frac{\lambda}{\tau} \int_{0}^{1} \zeta^{\lambda / \tau-1}(1-\zeta)^{n} d \zeta\right\} \\
= & -\frac{\lambda}{\tau^{2}} \int_{0}^{1} \zeta^{\lambda / \tau-1}(1-\zeta)^{n} d \zeta \\
& \quad-\frac{\lambda^{2}}{\tau^{3}} \int_{0}^{1} \zeta^{\lambda / \tau-1} \log (\zeta)(1-\zeta)^{n} d \zeta
\end{aligned}
$$

In the second integral, we substitute the following expansion of $\log (\zeta)$,

$$
\log (\zeta)=\log (1-(1-\zeta))=-\sum_{j=1}^{\infty} \frac{(1-\zeta)^{j}}{j}, \quad(|1-\zeta|<1)
$$

Then we have

$$
\frac{d}{d \tau} f_{n}(\tau, \lambda)=-\frac{1}{\tau} f_{n}(\tau, \lambda)+\sum_{j=1}^{\infty} \frac{1}{j} \frac{\lambda}{\tau^{2}} f_{n+j}(\tau, \lambda) .
$$

The following formulas are easily proved, and we omit the proof.

$$
\frac{1}{\tau} f_{n}(\tau, \lambda)=\frac{n}{\lambda} f_{n-1}(\tau, \lambda)-\frac{n}{\lambda} f_{n}(\tau, \lambda)
$$




$$
\begin{aligned}
& \frac{\lambda}{\tau^{2}} f_{n+j}(\tau, \lambda)=\frac{(n+j)(n+j-1)}{\lambda} f_{n+j-2}(\tau, \lambda) \\
&-\frac{(n+j)(2(n+j)-1)}{\lambda} f_{n+j-1}(\tau, \lambda) \\
&+\frac{(n+j)^{2}}{\lambda} f_{n+j}(\tau, \lambda) .
\end{aligned}
$$

Therefore by substituting these relations into (4.12), we have

$$
\begin{aligned}
\frac{d}{d \tau} f_{n}(\tau, \lambda)=\frac{n}{\lambda} & f_{n}(\tau, \lambda)-\frac{n}{\lambda} f_{n-1}(\tau, \lambda) \\
& +\sum_{j=1}^{\infty} \frac{1}{j} \frac{(n+j)(n+j-1)}{\lambda} f_{n+j-2}(\tau, \lambda) \\
& -\sum_{j=1}^{\infty} \frac{1}{j} \frac{(n+j)(2(n+j)-1)}{\lambda} f_{n+j-1}(\tau, \lambda) \\
& +\sum_{j=1}^{\infty} \frac{1}{j} \frac{(n+j)^{2}}{\lambda} f_{n+j}(\tau, \lambda) .
\end{aligned}
$$

Since $\partial u^{\theta}(\tau, z) / \partial \tau=\sum_{n=0}^{\infty} \phi_{n}(z) d f_{n}(\tau, \lambda) / d \tau$, we have

$$
\begin{aligned}
\frac{\partial u^{\theta}(\tau, z)}{\partial \tau}=\sum_{n=1}^{\infty} & \frac{n}{\lambda} \phi_{n}(z) f_{n}(\tau, z)-\sum_{n=0}^{\infty} \frac{n+1}{\lambda} \phi_{n+1}(z) f_{n}(\tau, z) \\
& +\sum_{n=0}^{\infty} \frac{(n+2)(n+1)}{\lambda}\left\{\sum_{j=1}^{n+2} \frac{\phi_{n+2-j}(z)}{j}\right\} f_{n}(\tau, \lambda) \\
& -\sum_{n=0}^{\infty} \frac{(n+1)(2 n+1)}{\lambda}\left\{\sum_{j=1}^{n+1} \frac{\phi_{n+1-j}(z)}{j}\right\} f_{n}(\tau, \lambda) \\
& +\sum_{n=0}^{\infty} \frac{n^{2}}{\lambda}\left\{\sum_{j=1}^{n} \frac{\phi_{n-j}(z)}{j}\right\} f_{n}(\tau, \lambda) .
\end{aligned}
$$

On the other hand, it is obvious that

$$
\frac{\partial^{2} u^{\theta}(\tau, z)}{\partial z^{2}}=\sum_{n=0}^{\infty} \phi_{n}^{\prime \prime}(z) f_{n}(\tau, \lambda) .
$$

Now by comparing the coefficients of $f_{n}(\tau, \lambda)(n=0,1,2, \ldots)$, we have the desired recurrence formula (4.11). In fact, it is sufficient to arrange the 
following relation which is obtained by comparing the coefficients of $f_{n}(\tau, \lambda)$ in the equation, $\partial u^{\theta} / \partial \tau=\partial^{2} u^{\theta} / \partial z^{2}$.

$$
\begin{aligned}
& \frac{n}{\lambda} \phi_{n}(z)-\frac{n+1}{\lambda} \phi_{n+1}(z) \\
& +\frac{(n+2)(n+1)}{\lambda}\left\{\phi_{n+1}(z)+\frac{\phi_{n}}{2}(z)+\cdots+\frac{\phi_{0}}{n+2}(z)\right\} \\
& \quad-\frac{(n+1)(2 n+1)}{\lambda}\left\{\phi_{n}(z)+\frac{\phi_{n-1}}{2}(z)+\cdots+\frac{\phi_{0}}{n+1}(z)\right\} \\
& \quad+\frac{n^{2}}{\lambda}\left\{\phi_{n-1}(z)+\frac{\phi_{n-2}}{2}(z)+\cdots+\frac{\phi_{0}}{n}(z)\right\}=\phi_{n}^{\prime \prime}(z) .
\end{aligned}
$$

In special cases where $n=1,2$, we have $\phi_{1}(z)=\lambda \varphi^{\prime \prime}(z)$ and

$$
\phi_{2}(z)=\frac{1}{2} \phi_{1}(z)+\frac{\lambda}{4} \phi_{1}^{\prime \prime}(z)=\frac{\lambda}{2} \varphi^{\prime \prime}(z)+\frac{\lambda^{2}}{4} \varphi^{(4)}(z) .
$$

This completes the proof.

\section{$\S 5$. New derivations of the heat kernel}

In this section, we shall give new methods to derive the heat kernel

$$
E(t, x)=\frac{1}{\sqrt{4 \pi t}} e^{-x^{2} / 4 t}, \quad x \in \mathbb{R}, t>0,
$$

by using the Borel sum of the following Cauchy problem,

$$
\frac{\partial u}{\partial \tau}=\frac{\partial^{2} u}{\partial z^{2}}, \quad u(0, z)=\frac{1}{z} .
$$

Here the formal solution $\hat{e}(\tau, z)$ is given by

$$
\hat{e}(\tau, z)=\sum_{n=0}^{\infty} \frac{(2 n) !}{n !} \frac{\tau^{n}}{z^{2 n+1}}=\frac{1}{\text { put }} \frac{\hat{f}}{z}\left(\frac{\tau}{z^{2}}\right) .
$$

We prove the following.

TheOREM 5.1. (i) For any $x \in \mathbb{R} \backslash\{0\}, \hat{e}(\tau, x)$ is Borel summable in a sector $-\pi / 2<\arg \tau<5 \pi / 2$, and let $e(\tau, x)$ be its Borel sum. Then we have

$$
\begin{aligned}
E(\tau, x)=\frac{-1}{2 \pi i}\left\{e \left(\tau e^{2 \pi i},\right.\right. & x)-e(\tau, x)\}, \\
& |\arg \tau|<\pi / 2, x \in \mathbb{R} \backslash\{0\}
\end{aligned}
$$


(ii) For $\tau$ with $|\arg \tau|<\pi / 2, \hat{e}(\tau, z)$ is finely Borel summable in both sides of the upper and lower half plane in complex $z$-plane. Let $e_{ \pm}(\tau, z)$ be the fine Borel sums in both half planes, respectively. Then we have

$$
E(\tau, x)=\frac{-1}{2 \pi i}\left\{e_{+}(\tau, x+i 0)-e_{-}(\tau, x-i 0)\right\}
$$

Proof. (i) (First Proof) Let $\hat{f}(\zeta)\left(\zeta=\tau / z^{2}\right)$ be the divergent power series defined in $(5.2)$, and let $g(\xi)$ be the formal Borel transform of $\hat{f}(\zeta)$, that is,

$$
g(\xi):=\sum_{n=0}^{\infty} \frac{(2 n) !}{(n !)^{2}} \xi^{n}=\frac{1}{\sqrt{1-4 \xi}} .
$$

This shows that $\hat{f}(\zeta)$ is Borel summable in the sector $-\pi / 2<\arg \zeta<5 \pi / 2$, and its Borel sum $f(\zeta)$ is obtained by analytic continuation of the following function.

$$
f^{\theta}(\zeta)=\frac{1}{\zeta} \int_{0}^{\infty e^{i \theta}} e^{-\xi / \zeta} \frac{d \xi}{\sqrt{1-4 \xi}}, \quad|\arg (\zeta)-\theta|<\pi / 2(0<\theta<\pi / 2) .
$$

Now we restrict $\zeta$ such that $|\arg \zeta|<\pi / 2$. Then we have

$$
f(\zeta)=\frac{1}{\zeta} \int_{0}^{1 / 4} e^{-\xi / \zeta} \frac{d \xi}{\sqrt{1-4 \xi}}+\frac{i}{\zeta} \int_{1 / 4}^{+\infty} e^{-\xi / \zeta} \frac{d \xi}{\sqrt{4 \xi-1}}
$$

and

$$
f\left(\zeta e^{2 \pi i}\right)=\frac{1}{\zeta} \int_{0}^{1 / 4} e^{-\xi / \zeta} \frac{d \xi}{\sqrt{1-4 \xi}}-\frac{i}{\zeta} \int_{1 / 4}^{+\infty} e^{-\xi / \zeta} \frac{d \xi}{\sqrt{4 \xi-1}} .
$$

Hence we have

$$
f\left(\zeta e^{2 \pi i}\right)-f(\zeta)=-\frac{2 i}{\zeta} \int_{1 / 4}^{+\infty} e^{-\xi / \zeta} \frac{d \xi}{\sqrt{4 \xi-1}}=-i \frac{\sqrt{\pi}}{\sqrt{\zeta}} e^{-1 / 4 \zeta} .
$$

Let $e(\tau, x)$ be the Borel sum of $\hat{e}(\tau, x)$ in the sector $-\pi / 2<\arg (\tau)<$ $5 \pi / 2$ for $x \in \mathbb{R} \backslash\{0\}$. Then

$$
e(\tau, x)=\frac{1}{x} f\left(\frac{\tau}{x^{2}}\right)
$$

and the desired formula (5.3) is obtained from (5.5). 
It is interesting to note the equations which $\hat{f}(\zeta)$ and $g(\xi)$ satisfy and this helps to explain the occurence of the hypergeometric function in the proof of theorem 3.1:

$$
\begin{aligned}
4 \zeta^{2} \hat{f}^{\prime \prime}(\zeta)+(10 \zeta-1) \hat{f}^{\prime}(\zeta)+2 \hat{f}(\zeta) & =0, \\
\xi(4 \xi-1) g^{\prime \prime}(\xi)+(10 \xi-1) g^{\prime}(\xi)+2 g(\xi) & =0,
\end{aligned}
$$

(Gauss' hypergeometric equation).

(Second Proof) Let $x \in \mathbb{R}$ be fixed, and $e(\tau, x)$ be the Borel sum of $\hat{e}(\tau, x)$ defined on $-\pi / 2<\arg (\tau)<5 \pi / 2$ which is obtained by the analytic continuation of the following function.

$e^{\theta}(\tau, x)=\frac{1}{\sqrt{4 \pi \tau}} \int_{\mathbb{R}-i \varepsilon} e^{-\zeta^{2} /(4 \tau)} \frac{d \zeta}{\zeta+x}, \quad|\arg (\tau)-\theta|<\pi / 2(0<\theta<\pi / 2)$,

where $\varepsilon>0$ is small and arbitrary.

We restrict $\tau$ such that $|\arg (\tau)|<\pi / 2$. Then we have

$$
e(\tau, x)-e\left(\tau e^{2 \pi i}, x\right)=\frac{1}{\sqrt{4 \pi \tau}} \oint_{|\zeta+x|=\delta} e^{-\zeta^{2} /(4 \tau)} \frac{d \zeta}{\zeta+x}=\frac{2 \pi i}{\sqrt{4 \pi \tau}} e^{-x^{2} /(4 \tau)},
$$

which implies the desired formula (5.3).

(ii) The formal Borel transform $v(s, z)$ of $\hat{e}(\tau, z)$ is given by $v(s, z)=$ $1 / \sqrt{z^{2}-4 s}$. Let $s>0$. Then $v(s, z)$ is univalent in $\mathbb{C} \backslash[-2 \sqrt{s}, 2 \sqrt{s}]$ and we have

$$
\frac{-1}{2 \pi i}\{v(s, x+i 0)-v(s, x-i 0)\}= \begin{cases}\frac{1}{\pi} \frac{1}{\sqrt{4 s-x^{2}}}, & x^{2}<4 s \\ 0, & x^{2} \geq 4 s\end{cases}
$$

We set $\Omega_{ \pm}:=\{\tau|| \arg \tau \mid<\pi / 2\} \times\{z \mid \pm \operatorname{Im} z>0\}$. Then the Laplace transforms $e_{ \pm}(\tau, z)$ of $v(s, z)$ are well defined in $\Omega_{ \pm}$by

$$
e_{ \pm}(\tau, z)=\frac{1}{\tau} \int_{0}^{\infty} e^{-s / \tau} \frac{d s}{\sqrt{z^{2}-4 s}}, \quad \pm \operatorname{Im} z>0 .
$$

The following asymptotic estimate is easily proved.

$$
\left|e_{ \pm}(\tau, z)-\sum_{n=0}^{N-1} \frac{(2 n) !}{n !} \frac{\tau^{n}}{z^{2 n+1}}\right| \leq C(\delta) K(z)^{N} N !|\tau|^{N}, \quad N=1,2,3, \ldots
$$


where $(\tau, z) \in\{|\arg \tau|<\pi / 2-\delta\} \times\{ \pm \operatorname{Im} z>0\}(\delta>0)$. Here $C(\delta)$ is a positive constant depending only on $\delta$, and $K(z)$ is a positive constant depending only on $z$. We remark that $K(z)$ can be taken uniformly on $z$ outside a bell type domain $\Delta(0 ; \varepsilon)$ (cf. (3.6)).

The expressions (5.8) imply the following relations.

$$
e_{+}(\tau, x+i 0)=\frac{1}{\tau} \int_{0}^{x^{2} / 4} e^{-s / \tau} \frac{d s}{\sqrt{x^{2}-4 s}}-\frac{i}{\tau} \int_{x^{2} / 4}^{\infty} e^{-s / \tau} \frac{d s}{\sqrt{4 s-x^{2}}}
$$

and

$$
e_{-}(\tau, x-i 0)=\frac{1}{\tau} \int_{0}^{x^{2} / 4} e^{-s / \tau} \frac{d s}{\sqrt{x^{2}-4 s}}+\frac{i}{\tau} \int_{x^{2} / 4}^{\infty} e^{-s / \tau} \frac{d s}{\sqrt{4 s-x^{2}}} .
$$

Now we get

$$
\frac{-1}{2 \pi i}\left\{e_{+}(\tau, x+i 0)-e_{-}(\tau, x-i 0)\right\}=\frac{1}{\tau \pi} \int_{x^{2} / 4}^{\infty} e^{-s / \tau} \frac{d s}{\sqrt{4 s-x^{2}}}=\frac{e^{-x^{2} / 4 \tau}}{\sqrt{4 \pi \tau}}
$$

which we want to prove.

\section{REFERENCES}

[Bal] W. Balser, From divergent power series to analytic functions, Lecture Notes in Mathematics 1582, Springer, Germany, 1994.

[Doe] G. Doetsch, Handbuch der Laplace-Transformation Vol II, Birkhäuser Verlag Basel und Stuttgart, Ulm, Germany, 1955.

[Ec] J. Ecalle, Les fonctions résurgentes I-III, Publication mathématiques d'Orsay, Paris, France, 1981, 1985.

[Ger-Tah] R. Gérard and H. Tahara, Singular nonlinear partial differential equations, Vieweg, 1996.

[Kow] S. Kowalevski, Zur Theorie der partiellen Differentialeichungen, J. Reine Angew. Math., 80 (1875), 1-32.

[Mal] N. Malgrange, Sommation des Séries divergentes, Expositiones Mathematicae, 13 (1995), Heidelberg, Germany, 163-222.

[Mal-Ram] B. Malgrange and J. P. Ramis, Fonctions multisommables, Ann. Inst. Fourier, 41 (1991), 1-16.

[Miy 1] M. Miyake, A remark on Cauchy-Kowalevski's theorem, Publ. Res. Inst. Sci., 10 (1974), 243-255.

[Miy 2] Global and local Goursat problems in a class of analytic or partially analytic functions, J. Differential Equations, 39 (1981), 445-463. 
[Miy 3] Newyon polygons and formal Gevrey indices in the Cauchy-GoursatFuchs type equations, J. Math. Soc. Japan, 41 (1991), 305-330.

[Miy-Has] M. Miyake and Y. Hashimoto, Newton polygons and Gevrey indices for partial differential operators, Nagoya Math. J., 128 (1992), 15-47.

[Miy-Yos 1] M. Miyake and M. Yoshino, Wiener-Hopf equation and Fredholm property of the Goursat problem in Gevrey space, Nagoya Math. J., 135 (1994), 165-196.

[Miy-Yos 2] — Fredholm property for differential operators on formal Gevrey space and Toeplitz operator method, C. R. Acad. Bulgare des Sci., 47 (1994), 21-26.

[Nev] F. Nevanlinna, Zur Theorie der asymptotischen Potenzreihen, Ann. Acad. Sci. Fenn. Ser. A1, Math. Dissertationes 12, 1918.

[Ōuc] S. Ōuchi, Characteristic Cauchy problems and solutions of formal power series, Ann. Inst. Fourier, 33 (1983), 131-176.

[Pic] E. Picard, Leọon sur quelques types simples d'équations aux dérivées partielles avec des applications à la physique mathématique, Gauthier-Villars, Paris, 1927.

[Ram 1] J. P. Ramis, Théorèmes d'indices Gevrey pour les équations différentielles ordinaire, Mem. Amer. Math. Soc. 48, No. 296, 1984.

[Ram 2] L Les séries k-sommables et leurs applications, Springer Notes in Physics, 126 (1980), 178-209.

[Was] W. Wasow, Asymptotic expansions for ordinary differential equations, Interscience Publ. John Wiley and Sons, Inc., New York, London, Sydney, 1965.

[Yon] A. Yonemura, Newton polygons and formal Gevrey classes, Publ. Res. Inst. Math. Sci., 26 (1990), 197-204.

D. A. Lutz

Department of Mathematical Science

San Diego State University

San Diego, CA, 92182-7720

USA

M. Miyake

Graduate School of Mathematics

Nagoya University

Nagoya, 464-8602

Japan

R. Schäfke

Départment de Mathématique

Université de Strasbourg

7, rue René-Descartes

67084, Strasbourg

France 